\title{
Supporting Information: \\ The Effects of Polyfluorene Polyelectrolyte Interfacial Layers on Selectivity and Recombination Measured Using the Interdigitated Back Contact Solar Cell
}

\author{
Christopher D. Weber, David P. Stay, and Mark C. Lonergan
}

\section{Details on Device Physics Simulations}

Numerical simulations were performed using the semiconductor module of COMSOL Multiphysics, Version 4.2. Below, we describe the system simulated, the equations solved in our specific implementation of the COMSOL semiconductor module, and details on the simulations performed. Table S1 lists the values of the simulation parameters used.

A one-dimensional model with position variable $x$ was used. As shown in Figure S1, it consisted of a line of silicon of length $l$ and with dopant (donor) density $N_{D}$. This region was contacted on one side with a Schottky contact (the contact of primary interest) and on the other with a silicon region of length $l_{2}$ that was either heavily n-doped $\left(\mathrm{n}^{+}\right) \mathrm{Si}$ with a dopant density $N_{D}^{+}$or heavily p-doped $\left(\mathrm{p}^{+}\right)$Si with a dopant density $N_{A}^{+}$. The heavily doped region was contacted with an Ohmic contact. The full set of parameters used in the simulations are listed in Table S1.

The default finite volume, Scharfetter-Gummel upwinding scheme in the COMSOL semiconductor module was used to discretized the drift/diffusion equations for the partial hole and electron currents, $J_{p}$ and $J_{n}$ :

$$
\begin{aligned}
& J_{n}=n \mu_{n} \mathcal{E}+\mu_{n} k T \frac{d n}{d x} \Pi\left(n / N_{c}\right) \\
& J_{p}=p \mu_{p} \mathcal{E}-\mu_{p} k T \frac{d p}{d x} \Pi\left(p / N_{v}\right)
\end{aligned}
$$

Here, $\mathcal{E}$ is the electric field; $k$ is the Boltzmann constant; $T$ is the temperature; $n$ and $p$ are the electron and hole concentrations; $\mu_{n}$ and $\mu_{p}$ are their mobilities; and $N_{c}$ and $N_{v}$ are the effective

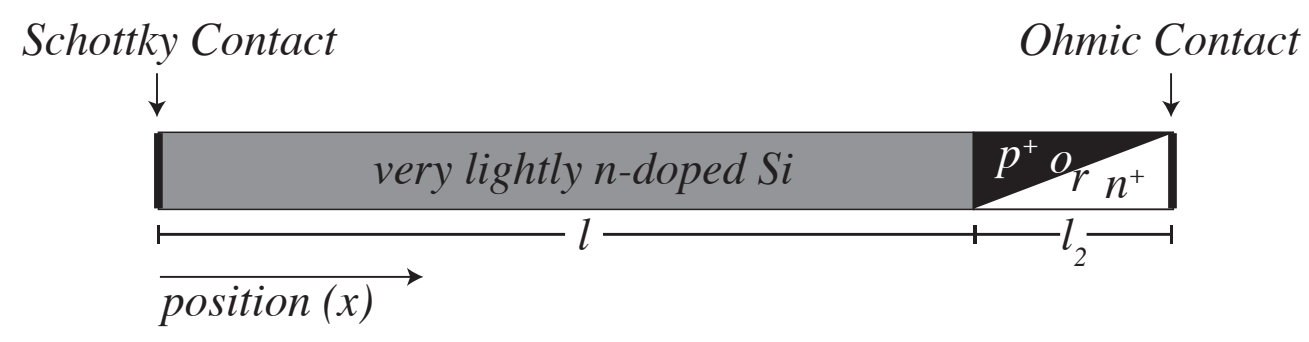

Figure S1: Schematic of simulation geometry. 
Table S1: Simulation model parameters

\begin{tabular}{|c|c|c|}
\hline Symbol & Description & Value \\
\hline$l$ & Si absorber thickness & $200 \mu m$ \\
\hline$l_{2}$ & Thickness of $\mathrm{n}^{+}$or $\mathrm{p}^{+}$contact & $20 \mu m$ \\
\hline$E_{g 0}$ & Silicon band gap at $T=0$ & $1.1785 \mathrm{eV}$ \\
\hline$E_{g}$ & Silicon band gap & $1.18 \mathrm{eV}-\left[\left(9.03 \times 10^{-5} \mathrm{eV} / \mathrm{K}\right) T+\left(3.05 \times 10^{-7} \mathrm{eV} / \mathrm{K}^{2}\right) T^{2}\right]$ \\
\hline$N_{C}$ & effective conduction band DOS & $2.8 \times 10^{19} \mathrm{~cm}^{-3}(T / 300 \mathrm{~K})^{3 / 2}$ \\
\hline$N_{V}$ & effective valence band DOS & $1.04 \times 10^{19} \mathrm{~cm}^{-3}(T / 300 \mathrm{~K})^{3 / 2}$ \\
\hline$\mu_{o, n}^{r e f}$ & $\mathrm{e}^{-}$mobility min. ref. & $1252 \mathrm{~cm}^{2} \mathrm{~V}^{-1} \mathrm{~s}^{-1}$ \\
\hline$\mu_{m i n, n}^{r e f}$ & $\mathrm{e}^{-}$mobility ref. & $88 \mathrm{~cm}^{2} \mathrm{~V}^{-1} \mathrm{~s}^{-1}$ \\
\hline$N_{o, n}^{r e f}$ & $\mathrm{e}^{-}$ref. impurity conc. & $1.26 \times 10^{17} \mathrm{~cm}^{-3}$ \\
\hline$\mu_{o, p}^{r e f}$ & $\mathrm{~h}^{+}$mobility min. ref. & $407 \mathrm{~cm}^{2} \mathrm{~V}^{-1} \mathrm{~s}^{-1}$ \\
\hline$\mu_{\text {min,p }}^{r e f}$ & $\mathrm{~h}^{+}$mobility ref. & $54.3 \mathrm{~cm}^{2} \mathrm{~V}^{-1} \mathrm{~s}^{-1}$ \\
\hline$N_{o, p}^{r e f}$ & $\mathrm{~h}^{+}$ref. impurity conc. & $2.35 \times 10^{17} \mathrm{~cm}^{-3}$ \\
\hline$\alpha_{o}^{r e f}$ & Arora $\alpha$ exponent & 0.88 \\
\hline$\beta_{1}$ & Arora $\beta_{1}$ exponent & -0.57 \\
\hline$\beta_{2}$ & Arora $\beta_{2}$ exponent & -2.33 \\
\hline$\beta_{3}$ & Arora $\beta_{3}$ exponent & 2.4 \\
\hline$\beta_{4}$ & Arora $\beta_{4}$ exponent & -0.146 \\
\hline$N_{D}$ & donor density in absorber & $1 \times 10^{13} \mathrm{~cm}^{-3}$ \\
\hline$N_{D}^{+}$ & dopant density in $\mathrm{n}^{+}$contact & $5 \times 10^{17} \mathrm{~cm}^{-3}$ \\
\hline$N_{A}^{+}$ & dopant density in $\mathrm{p}^{+}$contact & $5 \times 10^{17} \mathrm{~cm}^{-3}$ \\
\hline$\tau_{n}$ & SRH electron lifetime & $5 \mathrm{~ms}$ \\
\hline$\tau_{p}$ & SRH hole lifetime & $5 \mathrm{~ms}$ \\
\hline$C_{n}$ & Auger recomb. coeff. for $\mathrm{e}^{-}$ & $2 \times 10^{-30} \mathrm{~cm}^{6} \mathrm{~s}^{-1}$ \\
\hline$C_{p}$ & Auger recomb. coeff. for $\mathrm{h}^{+}$ & $2 \times 10^{-30} \mathrm{~cm}^{6} \mathrm{~s}^{-1}$ \\
\hline$\beta$ & radiative recomb. coeff. & $9.5 \times 10^{-15} \mathrm{~cm}^{3} \mathrm{~s}^{-1}$ \\
\hline$G_{n}$ & electron light generation rate & $1.2 \times 10^{19} \mathrm{~cm}^{-3} \mathrm{~s}^{-1}$ \\
\hline$G_{p}$ & hole light generation rate & $1.2 \times 10^{19} \mathrm{~cm}^{-3} \mathrm{~s}^{-1}$ \\
\hline$\phi_{n 0}$ & $\mathrm{e}^{-}$Schottky barrier $(T=0)$ & varied in simulation \\
\hline$\phi_{n}$ & $\mathrm{e}^{-}$Schottky barrier & $\phi_{n 0}-\left(E_{g 0}-E_{g}\right) /(2 q)$ \\
\hline$\phi_{p}$ & hole Schottky barrier & $E_{g} / q-\phi_{n}$ \\
\hline$A_{n}^{*}$ & Richardson constant for electrons & $110 \mathrm{~A} \mathrm{~K}^{-1} \mathrm{~cm}^{-2}$ \\
\hline$A_{p}^{*}$ & Richardson constant for holes & $90 \mathrm{~A} \mathrm{~K}^{-1} \mathrm{~cm}^{-2}$ \\
\hline$\nu_{n}$ & $e^{-}$charge transfer velocity & varied in simulation \\
\hline$\nu_{p}$ & $h^{+}$charge transfer velocity & varied in simulation \\
\hline
\end{tabular}


conduction and valence band densities of states. The form of eq S1 is for carriers governed by Fermi-Dirac statistics with the function $\Pi(\alpha)$ defined in terms of the Fermi-Dirac integrals $F_{j}$ of order $j=-1 / 2$ and $j=1 / 2$ :

$$
\begin{aligned}
\Pi(\alpha) & =\frac{\alpha}{F_{-1 / 2}\left(F_{1 / 2}^{-1}(\alpha)\right)} \\
F_{-\frac{1}{2}}(\eta) & =\frac{1}{-2 \sqrt{\pi}} \int_{0}^{\infty} \frac{\xi^{-1 / 2}}{1+e^{\xi-\eta}} d \xi \\
F_{\frac{1}{2}}(\eta) & =\frac{1}{\sqrt{\pi}} \int_{0}^{\infty} \frac{\xi^{1 / 2}}{1+e^{\xi-\eta}} d \xi
\end{aligned}
$$

An Arora mobility model was used for the mobilities in eq S1. Specifically, mobility $\mu$ was given by:

$$
\begin{gathered}
\mu=\mu_{\text {min }}+\frac{\mu_{o}}{1+\left(\frac{N}{N_{o}}\right)^{\alpha}} \\
\mu_{\text {min }}=\mu_{\text {min }}^{r e f}\left(\frac{T}{T_{\text {ref }}}\right)^{\beta_{1}} \\
\mu_{o}=\mu_{o}^{r e f}\left(\frac{T}{T_{r e f}}\right)^{\beta_{2}} \\
N_{o}=N_{o}^{r e f}\left(\frac{T}{T_{r e f}}\right)^{\beta_{3}} \\
\alpha=\alpha_{o}^{r e f}\left(\frac{T}{T_{r e f}}\right)^{\beta_{4}}
\end{gathered}
$$

where the values for the various Arora model parameters are given in Table S1.

The drift/diffusion eq S1 is solved together with Poisson's equation and the continuity equations. The former is given by:

$$
\epsilon_{r} \frac{d^{2} \phi}{d x^{2}}=q\left(p-n+N_{d}-N_{a}\right)
$$

where $q$ is the elementary charge, $\phi$ is the electrostatic potential, and $\epsilon_{r}$ is the relative dielectric constant. The continuity equations are:

$$
\begin{aligned}
& \frac{d n}{d t}=\frac{1}{q} \frac{d J_{n}}{d x}-U_{n} \\
& \frac{d p}{d t}=-\frac{1}{q} \frac{d J_{p}}{d x}-U_{p}
\end{aligned}
$$

Here, the $U_{n}$ and $U_{p}$ are the net recombination rates for electrons and holes, and they contain standard expressions for radiative, Shockley-Read-Hall and Auger recombination, as well as a generation term due to illumination. The recombination parameters used are listed in Table S1. A spatially invariant generation rate of electrons and holes by light was used (see Table S1). No 
difference was seen between this approach and a Beer-Lambert law for light generation owing to the long carrier diffusion lengths and geometry.

The boundary conditions for the contacts were as follows. The electron and hole partial currents at the boundary of the absorber layer with the Schottky contact were described by eqs 1 and 2 of the main manuscript, which are reproduced here:

$$
\begin{aligned}
& J_{n}=-q \nu_{n}\left(n_{s}-n_{s o}\right) \\
& J_{p}=q \nu_{p}\left(p_{s}-p_{s o}\right)
\end{aligned}
$$

where $n_{s}$ and $p_{s}$ are the electron and hole concentrations at the interface with the Schottky contacts, and $n_{s o}$ and $p_{s o}$ are their equilibrium values. The terms $\nu_{n}$ and $\nu_{p}$ are the charge transfer velocities of electrons and holes. Their maximum values $\nu_{n}^{*}$ and $\nu_{p}^{*}$ are typically observed at metal interfaces and are given by:

$$
\begin{aligned}
& \nu_{n}^{*}=\frac{A_{n}^{*} T^{2}}{q N_{c}} \\
& \nu_{p}^{*}=\frac{A_{p}^{*} T^{2}}{q N_{v}}
\end{aligned}
$$

where $A_{n}^{*}$ and $A_{p}^{*}$ are the Richardson constants defined in terms of thermionic emission theory under the assumption that carriers with energy greater than the interfacial barrier cross with unit probability. $N_{c}$ and $N_{v}$ are the effective conduction and valence band densities of states.

The boundary concentrations $n_{s o}$ and $p_{s o}$ in eq S9 are defined by quasi-equilibrium with the Fermi level in the contact:

$$
\begin{aligned}
& n_{s o}=N_{c} \exp \left(-\frac{q \phi_{n}}{k T}\right) \\
& p_{s o}=N_{v} \exp \left(-\frac{q \phi_{p}}{k T}\right)
\end{aligned}
$$

where $\phi_{n}$ and $\phi_{p}$ are the electron and hole barrier heights with $q\left(\phi_{p}+\phi_{n}\right)=E_{g}$. The barrier heights were assumed to follow the temperature dependence of the band gap $\left(E_{g}\right)$; the increase in $E_{g}$ with decreasing temperature was equally divided between $\phi_{n}$ and $\phi_{p}$ (see Table S1).

The Ohmic contact was modeled by assuming that the carrier concentrations at the boundary with the Ohmic contact were given by their equilibrium concentrations $n_{e q}$ and $p_{e q}$ :

$$
\begin{aligned}
& n_{e q}=N_{c} F_{\frac{1}{2}}\left(-\frac{E_{c}-E_{F}}{k T}\right) \\
& p_{e q}=N_{v} F_{\frac{1}{2}}\left(-\frac{E_{F}-E_{v}}{k T}\right)
\end{aligned}
$$

where $E_{F}$ is the Fermi level.

The boundary condition for the vacuum energy $\left(q V_{o}\right)$ relative to the reference equilibrium Fermi level in the semiconductor was taken to be:

$$
V_{o}=-\phi_{n}-\chi-V_{a p p}
$$


at the Schottky contact, and

$$
V_{o}=-\phi_{n}-\chi
$$

at the Ohmic contact.

The simulations were carried out as a function of $\nu_{n} / \nu_{n}^{*}, \nu_{p} / \nu_{p}^{*}$ and $\phi_{n 0}$, the electron barrier height at $T=0$. The simulation mesh consisted of 5000 mesh elements over the $200 \mu \mathrm{m}$ absorber and 500 mesh elements over the $20 \mu \mathrm{m}$ absorber. The mesh elements size was given by a symmetric geometric distribution proceeding from finer mesh elements at the interfaces to larger mesh elements in the bulk. Doubling the number of mesh elements did not affect the results. 
$2 V_{N}+V_{P}$ versus $V_{N}-V_{P}$ plot generated through variation of $\Delta \phi_{n p}$ versus variation of $\gamma_{\nu}$

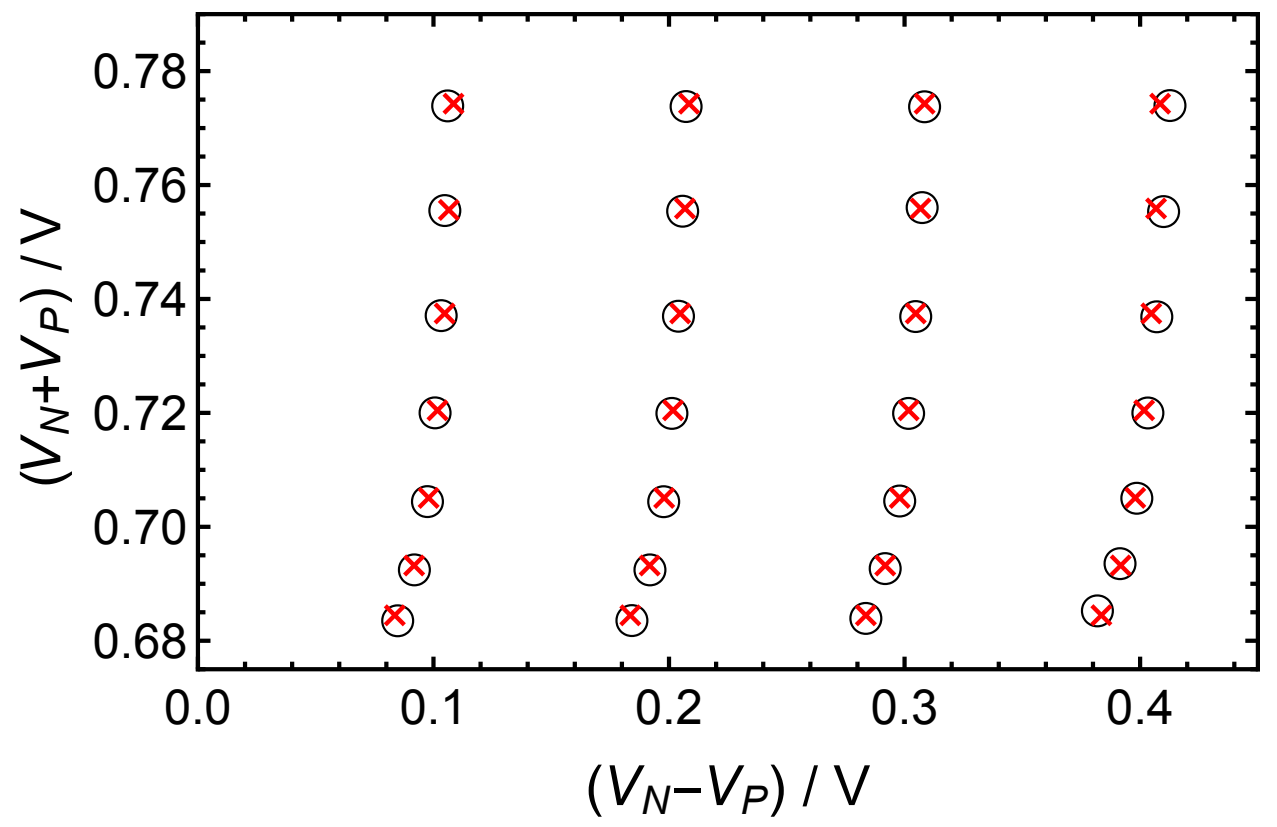

Figure S2: Comparison of simulated $V_{N}+V_{P}$ versus $V_{N}-V_{P}$ data generated in two ways. One set was generated holding $\gamma_{\nu}=100$ fixed while varying $\Delta \phi_{n p}($ red $\times)$ and corresponds to a subset of the data shown in Figure 4 of the main text. The other set was generated holding $\Delta \phi_{n p}=0.22 \mathrm{~V}$ and varying $\gamma_{\nu}$ (black 0 ). Each pair of points that nearly overlay have identical values of $J_{\text {on }}$ and $J_{o p}$.

\section{Symbol definitions}

Table S2 provides a complete list of symbol definitions used in the main body of the paper. 
Table S2: List of symbols and their definitions

\begin{tabular}{|c|c|}
\hline Parameter & Description \\
\hline$E_{F n}$ & electron quasi Fermi level \\
\hline$E_{F p}$ & hole quasi Fermi level \\
\hline$E_{F n}^{(s)}$ & electron quasi Fermi level at the semiconductor surface \\
\hline$E_{F p}^{(s)}$ & hole quasi Fermi level at the semiconductor surface \\
\hline$E_{g}$ & semiconductor band gap \\
\hline$k$ & Boltzmann constant \\
\hline$J$ & current density \\
\hline$J_{\text {on }}$ & electron equilibrium exchange current density at Schottky contact \\
\hline$J_{o p}$ & hole equilibrium exchange current density at Schottky contact \\
\hline$J_{o n}^{*}$ & electron equilibrium exchange current density in the metal limit \\
\hline$J_{o p}^{*}$ & hole equilibrium exchange current density in the metal limit \\
\hline$J_{n}$ & electron partial current density \\
\hline$J_{p}$ & hole partial current density \\
\hline$J_{s c}$ & short-circuit current density \\
\hline$N_{c}$ & conduction band effective density of states \\
\hline$N_{v}$ & valence band effective density of states \\
\hline$n_{s}$ & electron concentration at semiconductor surface at Schottky contact \\
\hline$n_{\text {so }}$ & equilibrium electron concentration at semiconductor surface at Schottky contact \\
\hline$p_{s}$ & hole concentration at semiconductor surface at Schottky contact \\
\hline$p_{\text {so }}$ & equilibrium hole concentration at semiconductor surface at Schottky contact \\
\hline$q$ & magnitude of elementary charge \\
\hline$R$ & interface recombination parameter \\
\hline$S_{h}$ & hole selectivity \\
\hline$S_{h, C D L}$ & hole selectivity in the contact determined limit \\
\hline$S_{e}$ & electron selectivity \\
\hline$T$ & temperature \\
\hline$V_{o c}$ & open-circuit voltage \\
\hline$V_{N}$ & open-circuit voltage measured between the $\mathrm{n}^{+}$contact and OSC \\
\hline$V_{P}$ & open-circuit voltage measured between the $\mathrm{p}^{+}$contact and OSC \\
\hline$\gamma_{\nu}$ & ratio of hole to electron charge transfer velocity (normalized by the thermal velocities) \\
\hline$\Delta \phi_{n p}$ & difference in electron and hole barrier heights \\
\hline$\nu_{n}$ & charge transfer velocity of electrons at Schottky contact \\
\hline$\nu_{p}$ & charge transfer velocity of holes at Schottky contact \\
\hline$\nu_{n}^{*}$ & thermal velocity of electrons in the semiconductor \\
\hline$\nu_{p}^{*}$ & thermal velocity of electrons in the semiconductor \\
\hline$\phi_{n}$ & electron barrier height at Schottky contact \\
\hline$\phi_{p}$ & hole barrier height at Schottky contact \\
\hline
\end{tabular}

